\title{
Nuclear Thermal Rocket Element Environmental Simulator (NTREES)
}

\author{
William J. Emrich, Jr. \\ NASA - Marshall Space Flight Center, M.S. ER24, Huntsville, Alabama 35812 \\ (256)544-7504,bill.emrich@nasa.gov
}

\begin{abstract}
To support the eventual development of a nuclear thermal rocket engine, a state-of-the-art experimental test setup has been constructed to evaluate the performance characteristics of candidate fuel element materials and geometries in representative environments. The test device simulates the environmental conditions (minus the radiation) to which nuclear rocket fuel components will be subjected during reactor operation. Test articles mounted in the simulator are inductively heated in such a manner as to accurately reproduce the temperatures and heat fluxes normally expected to occur as a result of nuclear fission while at the same time being exposed to flowing hydrogen. This project is referred to as the Nuclear Thermal Rocket Element Environment Simulator or NTREES. The NTREES device is located at the Marshall Space flight Center in a laboratory which has been modified to accommodate the high powers required to heat the test articles to the required temperatures and to handle the gaseous hydrogen flow required for the tests. Other modifications to the laboratory include the installation of a nitrogen gas supply system and a cooling water supply system. During the design and construction of the facility, every effort was made to comply with all pertinent regulations to provide assurance that the facility could be operated in a safe and efficient manner. The NTREES system can currently supply up to $50 \mathrm{~kW}$ of inductive heating to the fuel test articles, although the facility has been sized to eventually allow test article heating levels of up to several megawatts.
\end{abstract}

Keywords: Nuclear Thermal Rocket, Fuel Element, Testing

PACS: 28.50.Ky

\section{INTRODUCTION}

Missions to Mars will almost certainly require propulsion systems with performance levels exceeding that of today's best chemical engines. A strong candidate for that propulsion system is the Nuclear Thermal Rocket or NTR (Emrich and Young, 1992). Solid core NTR engines are expected to have specific impulses in excess of $850 \mathrm{sec}$ which is significantly greater than the best chemical lox/hydrogen engines which have maximum specific impulses in the range of $450 \mathrm{sec}$. NTR's operate by using a nuclear reactor to heat hydrogen propellant to high temperatures and expelling the resulting exhaust through a nozzle to produce thrust. In the late 1960's, a nuclear rocket program was instituted called Rover/NERVA which resulted in the construction of a number of prototypical nuclear engines. These engines in various tests produced up to 250,000 pounds of thrust and specific impulses up to $850 \mathrm{sec}$ with corresponding to fuel temperatures of about $2550 \mathrm{~K}$. (Finseth, 1991).

Since the last of the Rover/NERVA tests of the 1970's, NTR fuel development work has continued at a modest level to the present day. In particular, the former Soviet Union (Lutch) and the Innovative Nuclear Space Power and Propulsion Institute (INSPI) at the University of Florida have worked on new fuel forms which have the potential of performing considerably better than the earlier particle matrix and composite fuels which suffered from various hot hydrogen related corrosion effects (Taub, 1975), (Koenig, 1986). These fuels are comprised primarily of solid solution uranium tricarbides incorporating gradient coatings for enhanced protection against hydrogen corrosion. Recent studies indicate that these fuels have the potential of operating at temperatures exceeding $3000 \mathrm{~K}$ for several hours (Knight and Anghaie, 1999). 
Testing of the Rover/NERVA engines was performed in the Nevada desert by allowing the hot hydrogen propellant exhaust to escape directly into the atmosphere. Such testing would likely not be permitted in today's regulatory environment. These tests, while impressive for what they accomplished, nevertheless, did not allow for the detailed examination of fuel behavior during reactor operation. As a result, it was quite difficult to observe the mechanisms which led in certain instances to fuel failure. Since these tests showed that little fuel damage was the result of radiation effects, it should be possible to conduct small scale non nuclear tests of single fuel elements or even partial elements which could fill in many important data gaps that still exist. Fuel elements in such tests could be heavily instrumented and their behavior observed under a variety of normal and off normal conditions. Such testing would provide valuable information with regard to optimizing fuel element geometries to minimize the effects of hot hydrogen corrosion and assuming sufficiently high gas temperatures are attained also take advantage of performance gains resulting from hydrogen dissociation. The electrical heating and hydrogen flow requirements for such tests are quite modest and the results of such experiments could yield considerable insight into fuel behavior under extreme conditions.

The primary objective of this paper is to describe the general characteristics of an experimental facility which has been built at the Marshall Space Flight Center to permit the testing and characterization of candidate nuclear fuel materials at high temperatures and heat fluxes especially with regard to their durability and resistance to attack by flowing hot hydrogen. Experiments would involve applying direct resistance or inductive heating to test articles to simulate the temperatures and heat fluxes which would occur as a result of nuclear fission. Because the heating is electrical rather than nuclear, the problems associated with fission product contamination and radiation is avoided. The test data resulting from these experiments would be used to develop wall heat transfer and momentum loss correlations which could be used to determine realistic performance characteristics of NTR engines.

\section{SYSTEM OVERVIEW}

To support the on-going nuclear thermal propulsion effort, a state-of-the-art experimental test setup has been constructed to evaluate the performance characteristics of candidate fuel element materials and geometries in representative environments. This device can simulate the environmental conditions (minus the radiation) to which nuclear rocket fuel components will be subjected during reactor operation. Test articles mounted in the simulator are inductively heated in such a manner so as to accurately reproduce the temperatures and heat fluxes which would normally occur as a result of nuclear fission and would be exposed to flowing hydrogen. The project to perform this testing is referred to as the Nuclear Thermal Rocket Element Environment Simulator (NTREES). The NTREES device is located in the building 4205 laboratory 110 high-bay. The laboratory has been modified to accommodate the high powers required to heat the test articles to the required temperatures and to handle the gaseous hydrogen flow required for the tests. Other modifications to the high bay were necessary to supply nitrogen gas and cooling water to the system. With regard to the hydrogen flow requirement, every effort has been made to comply with all pertinent regulations so as to guarantee that the facility can be operated in a safe and efficient manner.

\section{Nuclear Thermal Rocket Element Environmental Simulator (NTREES)}

To support the on-going nuclear thermal propulsion effort, a state-of-the-art experimental test setup is required to evaluate the performance characteristics of candidate fuel element materials and geometries in representative environments. Test articles mounted in the simulator will be inductively heated in such a manner so as to accurately reproduce the temperatures and heat fluxes which would normally occur as a result of nuclear fission and would be exposed to flowing hydrogen. By heating test articles electrically rather than in a nuclear test reactor, most of the relevant data with regard to the test element's operational characteristics can be obtained without the cost and long term planning required for nuclear testing.

The scale of the project is such that if required, fuel elements at least the size of those used in the NERVA tests can eventually be accommodated within the facility. The current power levels in the facility will not yet support testing of this magnitude, however. The project to perform this testing is referred to as the Nuclear Thermal Rocket Element Environment Simulator (NTREES). The basic laboratory setup for NTREES is illustrated in Figure 1. The NTREES capabilities are summarized in Table 1. 


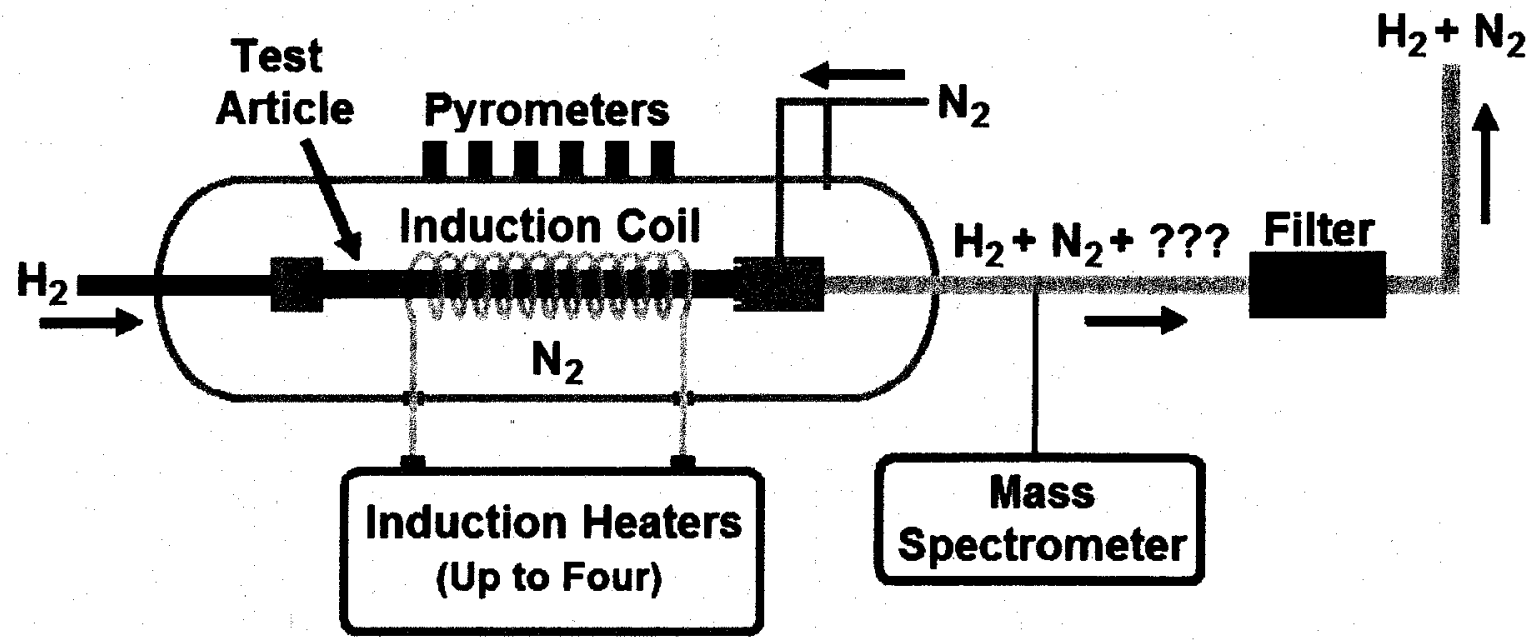

FIGURE 1. NTR Element Environmental Simulator Operational Layout.

TABLE 1. NTREES Operational Characteristics.

\begin{tabular}{ll}
\hline Operational Parameter & Value \\
\hline Maximum Test Article Temperature (K) & $>3000$ \\
Maximum Hydrogen Flow Rate (gm/s) & 35 \\
Maximum Nitrogen Flow Rate (gm/s) & 1800 \\
Operating Pressure Range (MPa) & Vacuum to 7 \\
Current Power Capability (kw) & Up to 50 \\
Maximum Expandable Power Capability (kw) & Up to 3500 \\
Maximum Test Article Length (m) & 2.50 \\
Maximum Test Article Diameter (m) & 0.30 \\
\hline
\end{tabular}

\section{Vacuum/Pressure Chamber}

The NTREES vacuum/pressure chamber consists of a water cooled ASME code stamped vessel and its associated support stand. The vessel has a maximum operating pressure of $6.9 \mathrm{MPa}$ and a design pressure of $7.6 \mathrm{MPa}$. During ASME qualification testing the vessel withstood pressures $30 \%$ over design pressure (9.9 MPa). In size the vessel is approximately $305 \mathrm{~cm}$ long and $91 \mathrm{~cm}$ diameter. The central chamber without the heads weighs about $4435 \mathrm{~kg}$ and the total weight of the chamber with the heads is about $7650 \mathrm{~kg}$. Much effort was spent designing flexibility into the vessel so that it would be capable of testing a wide variety of NTR fuel elements and other components. The chamber has 21 small $5.1 \mathrm{~cm}$ diameter sapphire viewports situated along the length of the vessel to accommodate pyrometers which remotely measure the temperatures distributions in the components being tested. Also along the length of the vessel are 9 somewhat larger $7.6 \mathrm{~cm}$ diameter viewports which allow test personnel a means to visually observe the condition of the test fixture during the course of testing.

In addition, the chamber head assemblies have single $7.6 \mathrm{~cm}$ diameter viewports to allow visual access down the axial length of the chamber. Five large $30.5 \mathrm{~cm}$ penetrations along the bottom of the chamber are included to accommodate power feedthroughs for the induction heaters, although one of the penetrations could also be adapted for use as part of a vacuum system installation. To allow hand access to the interior of the chamber when the heads 
are attached, there are four $15.2 \mathrm{~cm}$ penetrations along each side of the lower diagonal of the chamber. These penetrations allow work to be performed on the test article and coil assembly after they are mounted in the chamber without necessitating the time consuming and expensive job of removing the head assemblies. Along the top of the chamber are ten $5.1 \mathrm{~cm}$ penetrations which are designated as "spares" to accommodate additional instrumentation or systems as needed.

\section{Exhaust Treatment System (ETS)}

The Exhaust Treatment System or ETS is used in NTREES to process the effluent stream of hydrogen and nitrogen as it leaves the main NTREES chamber. The ETS is, in reality, three separate systems which work in tandem to cool and filter the effluent stream so that it may be safely discharged into the environment. The three systems include the hydrogen/nitrogen mixer assembly which is partially located within the main NTREES chamber, the water to gas heat exchanger, and the filter/backpressure system. The entire Exhaust Treatment System is mounted on a support stand and connected to the NTREES pressure chamber at the end support flange. Figure 2 illustrates the ETS as configured on the main NTREES chamber. In addition to the systems just enumerated, additional filtering components will be required in the pressure relief system and bleed lines.

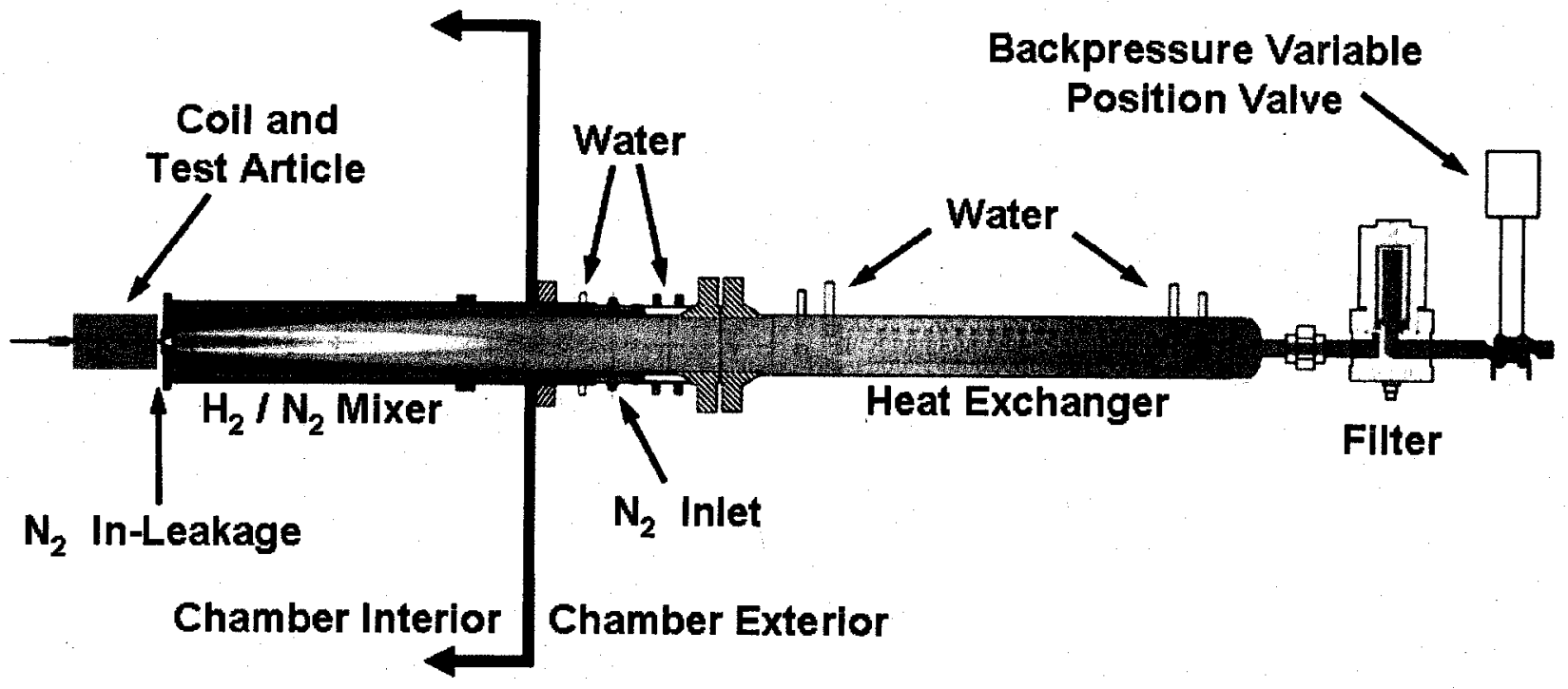

FIGURE 2. Exhaust Treatment System.

The NTREES mixer assembly is used to support the fuel material test pieces during testing while they are being heated and exposed to flowing hydrogen. In addition to supporting the test pieces, the mixer assembly dilutes and cools the hot hydrogen as it leaves the NTREES with large quantities of high pressure, room temperature nitrogen.

Nitrogen is injected into the mixer assembly through taps on the outside of the chamber and is distributed within the mixer such that the hot hydrogen is prevented from touching the sides of the mixer until it has been well diluted and cooled by the nitrogen. To provide additional temperature margins, the entire mixer assembly is internally cooled with water flowing at about $0.0035 \mathrm{~m}^{3} / \mathrm{s}$.

To eliminate the need for a high temperature seal at the test piece / mixer assembly interface, the chamber nitrogen pressure is kept slightly above the hydrogen pressure in the test piece. In-leakage of nitrogen through a small gap in the test article support cap cools the interface where the cap supports the test piece and prevents significant quantities of hydrogen from escaping into the chamber. Nevertheless, because the test article support cap still experiences fairly high temperatures at the points where the fixture physically touches the test article, it was deemed prudent to construct the cap from a high temperature alloy (in the present case a molybdenum/rhenium alloy) to preclude the possibility of melting portions of the fixture during operation. 


\section{Induction Heater}

The induction heater used to simulate fission processes in the test articles is a $50 \mathrm{~kW}, 50 \mathrm{kHz}$ unit fabricated by Inductoheat Corporation. Figure 3a illustrates the induction power supply and Figure $3 \mathrm{~b}$ illustrates the induction coil. In the figure, an insulating sleeve has been removed from the coil to show the test article in its test position. It is anticipated that for most tests, the insulating sleeve will be necessary to prevent excessive radiation losses from the test article and to protect the coil windings. The coil and test article are mounted in the pressure chamber and are energized by the induction heater located just outside the chamber. Connections are made via large feedthroughs mounted underneath the chamber. Cooling for the induction heater is accomplished with a dedicated cooling water and heat exchanger system which is connected to the building service water system

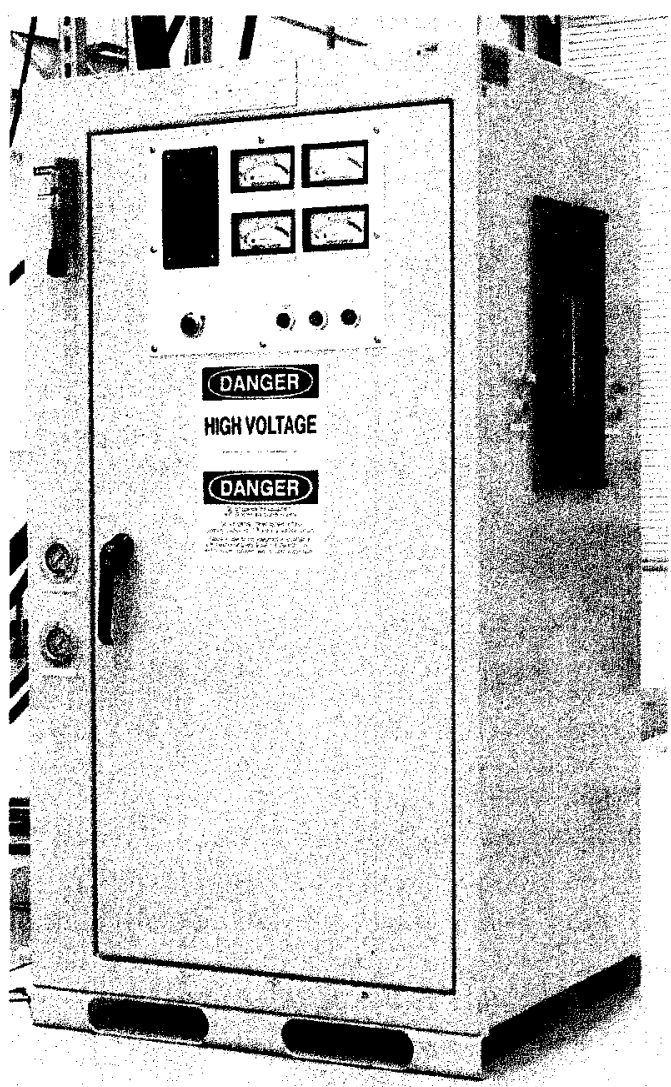

(a) Induction Heater.

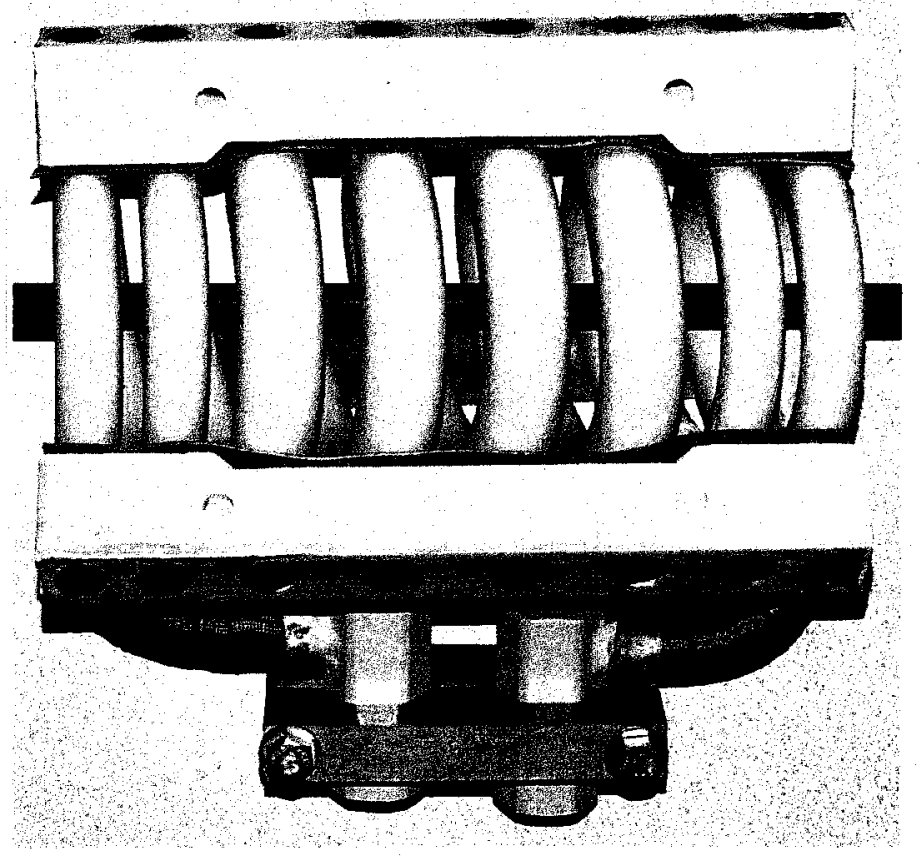

(b) Coil Assembly.

FIGURE 3. Induction Heater System.

\section{Data Acquisition System}

The NTREES experiment is equipped with a variety of hardware for instrumentation, control, and data acquisition. The hardware are distributed around the test chamber and support equipment, but integrated and controlled from one piece of software running on one computer located in the control room.

The instrumentation on NTREES includes:
- $\quad$ Pressure sensors for $\mathrm{GH}_{2}$ and $\mathrm{GN}_{2}$
- Temperature sensors for $\mathrm{GH}_{2}$ and $\mathrm{GN}_{2}$
- $\quad$ Flow sensors for $\mathrm{GH}_{2}$ and $\mathrm{GN}_{2}$
- Thermocouples for general temperature measurements
- Hydrogen detection for the test chamber and room 110 
- Mass spectrometer to measure gas composition

Pyrometers are used for high-temperature, non-contact measurement of the test article temperature. They operate on the principle that a body emits radiation in accordance with its temperature. The radiation that is emitted is highly dependent upon the emissivity of the body. The emissivity is dependent upon material composition, surface finishing, and temperature, among other things.

The exit gas composition will be measured with a mass spectrometer. The spectrometer chosen for NTREES has a large AMU (atomic mass unit) range to be able to see potential uranium compounds. The unit also has a gas sampling system that acts as a mechanical multiplexer; any one of up to 12 plumbed inputs can be directed to the spectrometer. Different selections can be made during a test run to determine how gas composition changes at various locations. By measuring the gas composition of gas stream, real time estimates may be made of test article erosion

\section{NTREES SYSTEM SAFETY}

During the NTREES design and buildup phases, every effort was made to meet or exceed all relevant safety standards, which include those of NFPA (National Fire Protection Association), OSHA (Occupational Safety \& Health Administration), and NASA Safety. In addition, numerous reviews were initiated whereby outside groups were given the opportunity to critique the NTREES design so as to provide additional confidence that no flaws in the design or operation of the system would compromise personnel safety.

The design highlights relating to NTREES hydrogen safety are:

- Welded tubing inside the building except where connections are necessary

- Fail safe system design (e.g. hydrogen shutoff valve fails to isolate hydrogen source external to building)

- Use of nitrogen purges to remove air

- Hydrogen detection at key locations around facility and in test chamber

- Class I, Division II Group B (hazardous) classification around $\mathrm{GH}_{2}$ inlet manifold and main process pressure-control valve

- Hazardous classification (above) rating met by use of explosion-proof hardware for all ignition sources, which have been minimized

- All hardware required in the Div II zone is placed away from possible ignition sources

- Use of a real-time system controller that independently shuts off GH2 flow in the event of:

- detection of a hydrogen leak or an unacceptable hydrogen level in the chamber

- loss of purge pressure or flow

- test article rupture

- possible failure in any safety monitoring system

The entire NTREES facility is illustrated in Figures 4 and 5.

\section{SUMMARY}

For an NTR to operate at maximum efficiency the nuclear fuel comprising the core must operate safely at the highest feasible temperatures for at least several hours. Presently, the fuel forms and materials most appropriate for use in NTR systems in terms of their robustness and durability are not clearly defined, and considerable testing will be required to develop a database adequate for making intelligent decisions on appropriate nuclear fuel designs. The NTR environmental simulator will fulfill the testing needs for many of the fuel types presently under consideration and will allow researchers to develop the fuels database ultimately required for informed decision making. In particular, the NTR environmental simulator will allow detailed observations to be made of fuel failure mechanisms in test articles especially with regard to corrosion rates as functions of propellant temperature 


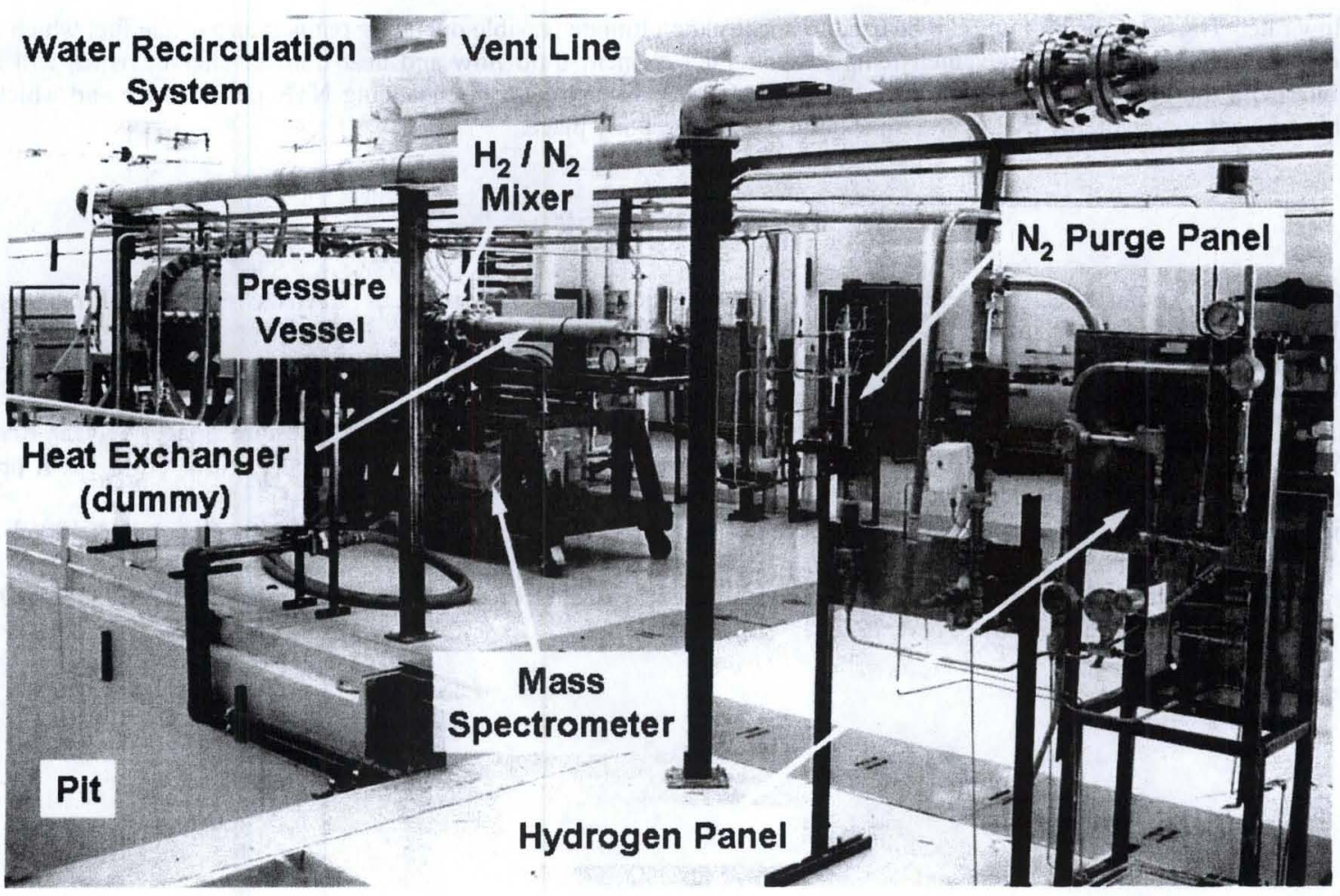

FIGURE 4. NTREES Front Side.

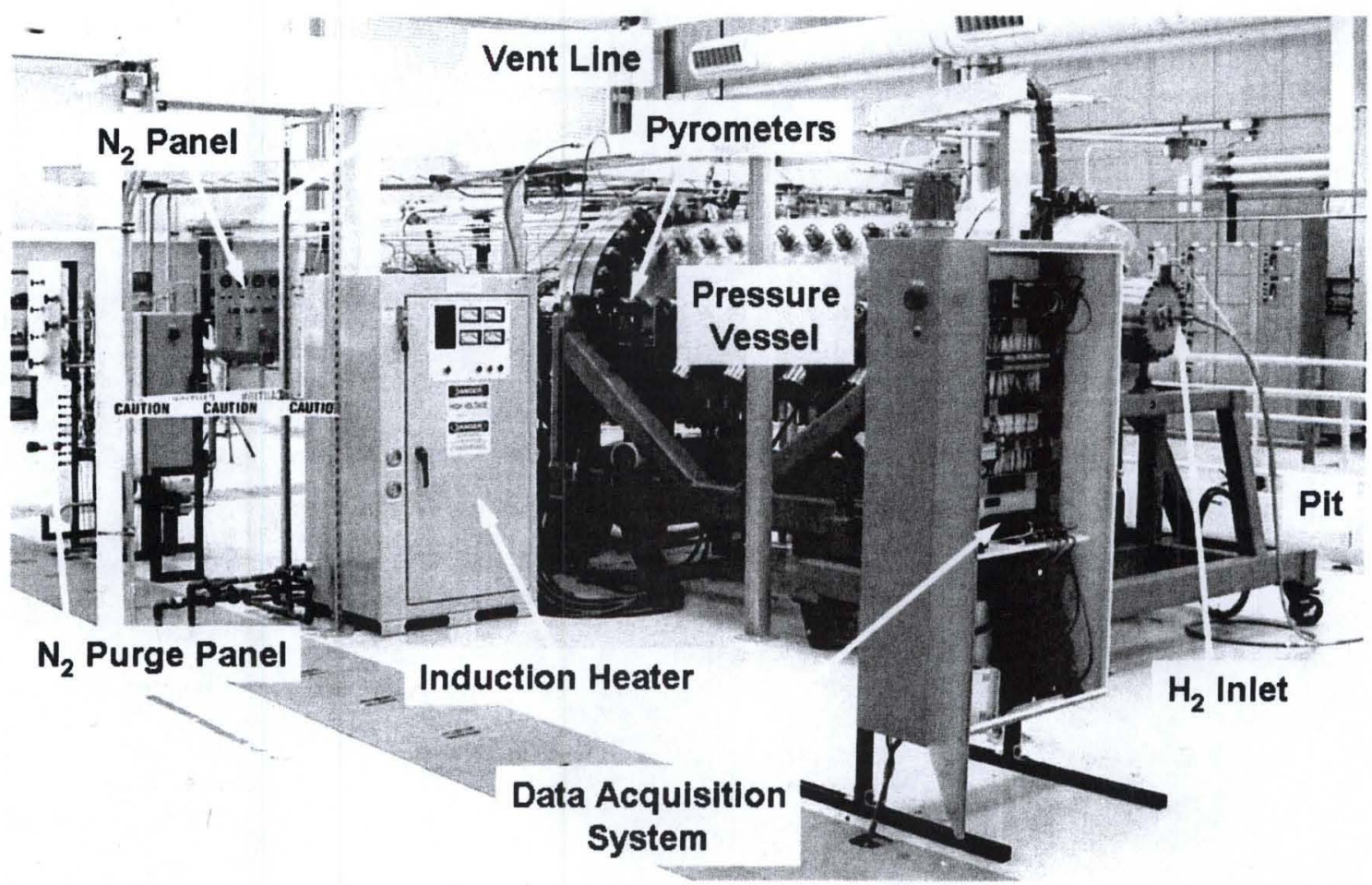

FIGURE 5. NTREES Back Side. 
and flow rate. These corrosion rates can be used to specify the ultimate feasible operating temperature of the fuel which may be considerably different from the fuel melt temperature. Fuel element fluid flow and heat transfer characteristics will also be examined with the goal being to determine which designs are most efficient in enhancing NTR performance and which could possibly take advantage of any hydrogen dissociation processes taking place.

\section{REFERENCES}

Emrich, W. J. and Young A. C., "Nuclear Propulsion System Options for Mars Missions, "AIAA Space Programs and Technologies Conference, AIAA 92-1496, Huntsville, AL, 1992.

Finseth, J.L., "Rover Nuclear Rocket Engine Program: Overview of Rover Engine Tests, Final Report," Contract NAS 8-37814, NASA, Marshall Space Flight Center, Science and Engineering, 1991.

Knight, T. and Anghaie, S., "Processing Of Pseudo-Ternary Carbide Fuels For High Temperature Space Nuclear Reactors," STAIF 1999, edited by Mohamed S. El-Genk, AIP Conference Proceedings 458, Woodbury, New York, 1999, pp 1487. 1494.

Koenig, D., "Experience Gained from the Space Nuclear Rocket Program (Rover)," Los. Alamos Report LA-10062-H, Los Alamos National Laboratory, Los Alamos, NM, 1986.

Taub, J. M., "A Review of Fuel Element Development for Nuclear Rocket Engine," Los Alamos Report LA-5931, Los Alamos National Laboratory, Los Alamos, NM, 1975. 